\title{
Casp8 hypomethylation and neural tube defects in association with polycyclic aromatic hydrocarbon exposure
}

Yun Huang ${ }^{1,2}$, Aiguo Ren ${ }^{1,2^{*}}$, Linlin Wang ${ }^{1,2^{*}}$, Lei Jin ${ }^{1,2}$, Shanshan Lin ${ }^{1,3}$, Zhiwen Li ${ }^{1,2}$ and Jasmine A. McDonald $d^{4,5}$

\begin{abstract}
Background: Epidemiological studies have found that prenatal exposure to polycyclic aromatic hydrocarbons (PAHs) is associated with increased risk for neural tube defects (NTDs). Aberrant DNA methylation, excessive apoptosis, and oxidative stress have been implied as the mechanism underlying the association between PAH exposure and NTDs, respectively. However, the role of DNA methylation aberration of apoptotic initiator CASP8 (caspase-8, apoptosis-related cysteine peptidase) in the formation of NTDs in association with PAH exposure is not known. By combining a case-control study and mouse model, we aimed to explore the full spectrum of the links from PAH exposure, oxidative stress, CASP8 methylation change, caspase-8 activation, apoptosis, to NTD formation.

Results: Hypomethylation of CASP8 promoter was noticed in the microarray profiled by Infinium HumanMethylation450 BeadChip using neural tissues from 10 terminated NTD fetuses and 8 terminated nonmalformed fetuses (14 CpG sites, with $\beta$ difference ranging between 8.8 and 26.3\%), and was validated in a larger case-control sample performed with neural tissues from 80 NTD cases and 32 non-malformed fetuses, using the Sequenom MasSARRAY system (7 CpG sites). Hypomethylation of CASP8 was a risk factor for NTDs (aOR $=1.11 ; 95 \%$ $\mathrm{Cl}, 1.05-1.17)$ based on the logistic regression model. According to Pearson's correlation, methylation levels of CASP8 were inversely correlated with PAH concentrations in maternal serum and with oxidative stress markers in fetal neural tissues $(p<0.05)$. In the animal study, increased NTD rates (13.5\% frequency), Casp 8 hypomethylation, caspase-8 upregulation, increased caspase-8 cleavage, and excessive apoptosis were found in mouse embryos cultured with benz(a)pyrene (BaP) in vitro. Antioxidant N-acetyl-L-cysteine (NAC) and BaP co-treatment attenuated the changes found in $\mathrm{BaP}$ treatment group.
\end{abstract}

Conclusions: Hypomethylation of Casp8 promoter is associated with the formation of NTDs, and Casp8 hypomethylation may be induced by oxidative stress that resulted from exposure to PAHs.

Keywords: Neural tube defects, Polycyclic aromatic hydrocarbons, Caspase-8, Hypomethylation, Apoptosis, Oxidative stress

\section{Introduction}

Neural tube defects (NTDs) are a group of severe congenital malformations of the central nervous system resulting from incomplete closure of the neural tube during early embryogenesis [1]. On average, NTDs affect approximately 1 in every 1000 established pregnancies

\footnotetext{
* Correspondence: renag@bjmu.edu.cn; linlinwang@bjmu.edu.cn ${ }^{1}$ Institute of Reproductive and Child Health, National Health Commission Key Laboratory of Reproductive Health, Peking University, Beijing 100191, China
} Full list of author information is available at the end of the article worldwide, with a higher prevalence in miscarried pregnancies [2, 3]. Annually, more than 320,000 infants are born with NTDs around the world [4]. The etiology of NTDs is complex, with contributions from environmental exposure, genetic variants, and gene-environment interactions. It is suggested that maternal exposure to polycyclic aromatic hydrocarbons (PAHs), a class of ubiquitous organic persistent pollutants, is associated with an increased risk for NTDs in humans [5-8], and an elevated occurrence of NTDs is also found in mouse embryos of dams exposed to benzo(a)pyrene (BaP), a well-studied PAH [9]. Although pathological processes, 
including oxidative stress $[9,10]$, DNA adduct formation [7], and DNA methylation dysregulation [11], are reported to be involved in PAH teratogenicity, the underlying mechanisms that cause PAH-induced NTDs remain unknown.

Spatiotemporally regulated apoptosis is a requirement for normal neural tube closure [12]. Excessive apoptosis has been found in fetal central nervous tissue of human NTD cases and murine NTDs [9, 13, 14], which indicate a role for un-regulated apoptosis in NTD formation. The activation of aspartate-specific cysteine protease (caspase)-8 acts as an initiator in the extrinsic apoptosis pathway which is initiated upon activation of death receptors [15]. Both studies in human and mice have revealed that levels of cleaved caspase- 8 are significantly higher in neural tissue of NTD cases relative to controls $[13,16]$. As reviewed in Das and Henkler's article, PAH-mediated genotoxicity can trigger extrinsic apoptosis, with increases in caspase-8 $[17,18]$. In previous studies, we have identified increased levels of apoptotic cells and cleaved caspase- 8 in the neural tissue of NTD cases, and the percentage of apoptotic cells in fetal neural tissue was positively correlated with the concentrations of PAHs in maternal serum $[9,13]$. However, it remains unknown whether excessive apoptosis is caused by exposure to PAHs via caspase- 8 dysregulation.

Growing evidence has indicated that PAHs may result in DNA methylation dysregulation $[19,20]$, the best-known epigenetic change thought to be involved in the development of many multifactorial diseases, including NTDs [11, 21]. Exposure to PAHs leads to oxidative stress $[9,22]$, and oxidative stress has been reported to be associated with DNA hypomethylation, as found in human, animal, and cell studies [23-25]. A mouse study suggested that reactive oxygen species (ROS) may induce hypomethylation and subsequent upregulation of specific genes [26]. Increased levels of caspase- 8 and ROS have both been detected in hyperglycemia-induced NTDs, and antioxidants could ameliorate NTD occurrence with decreased caspase-8 cleavage $[14,16]$. In addition, ROS generation induced by PAHs has been identified as a mechanism of PAH-mediated apoptosis [27]. However, the full spectrum of the links from PAH exposure, oxidative stress, CASP8/Casp 8 methylation change, caspase- 8 activation, apoptosis, to NTD formation has not yet been fully explored.

Therefore, we hypothesized that the decreased methylation of caspase- 8 gene (Casp8), in association with oxidative stress induced by PAH exposure, may lead to NTDs. We tested this hypothesis by examining the methylation levels of CASP8 in neural tissues from a small sample of NTD cases and controls, using a genomic microarray. Then, the discovered site-locus-specific methylation was validated using neural tissues from a larger sample of
NTD cases and non-malformed controls. The correlations between the validated DNA methylation intensity of CASP8 with PAH concentrations in maternal serum and with oxidative stress markers in fetal neural tissues were examined. Finally, to verify the findings in the human subject study, the CpG sites of Casp8, expression of caspase-8, and apoptosis levels were examined in mouse embryos exposed to $\mathrm{BaP}$ or $\mathrm{BaP}$ and antioxidant $N$-acetyl-L-cysteine (NAC) co-treatment in an in vitro whole-embryo culture model.

\section{Results}

\section{CASP8 methylation in microarray data}

In the discovery stage, the methylation values of $\mathrm{CpG}$ sites in CASP8 were extracted from the genome-wide methylation dataset generated using an HM450K microarray. A total of $26 \mathrm{CpG}$ sites in CASP8 were detected with the microarray, of which 22 were more hypomethylated in NTD cases compared to controls. Of these 22 sites, 14 were enriched in the promoter region and were significantly hypomethylated in NTD fetuses. For those 14 CpG sites, six CpG probes (cg09464206, cg27410837, cg04048517, cg24410214, cg19448993, and cg02878216) were located in the TSS1500 region, four (cg23882545, cg14962032, cg25748441, and cg25095814) were located in the TSS200 region, and the remaining four (cg00978584, cg20418725, cg12604794, and cg04286206) were located at 5'UTR of CASP8 (Additional file 1: Table S4).

\section{Validation of CASP8 methylation in a larger sample}

In the validation stage, we examined the methylation status of CpG sites in the promoter region of CASP8 identified in the discovery stage using Sequenom EpiTYPER in 80 NTD cases and 32 controls. The distribution of the maternal and fetal characteristics is presented in Additional file 1: Table S5. Significant differences were found in maternal education, occupation, parity, unplanned pregnancy, and periconceptional folate supplementation between the two groups. Case mothers were more likely to be less-educated farmers, to have higher parity, and to have had periconceptional folate supplementation than control mothers. A significantly lower proportion of case women (38.0\%) reported unplanned pregnancies, compared to $74.2 \%$ of control women. No significant differences were found between the case and control groups for any other characteristics.

We designed two DNA amplicons to validate the CpG sites discovered in stage one (Fig. 1a). Significantly hypomethylated $\mathrm{CpG}$ sites with a $\beta$ difference (absolute value) greater than 0.2 were chosen as a priority for DNA amplicon design. Amplicon 1 covered three $\mathrm{CpG}$ sites (CpG1 to CpG3), in which CpG3 was the same as the hypomethylated CpG site of cg25748441 identified in 

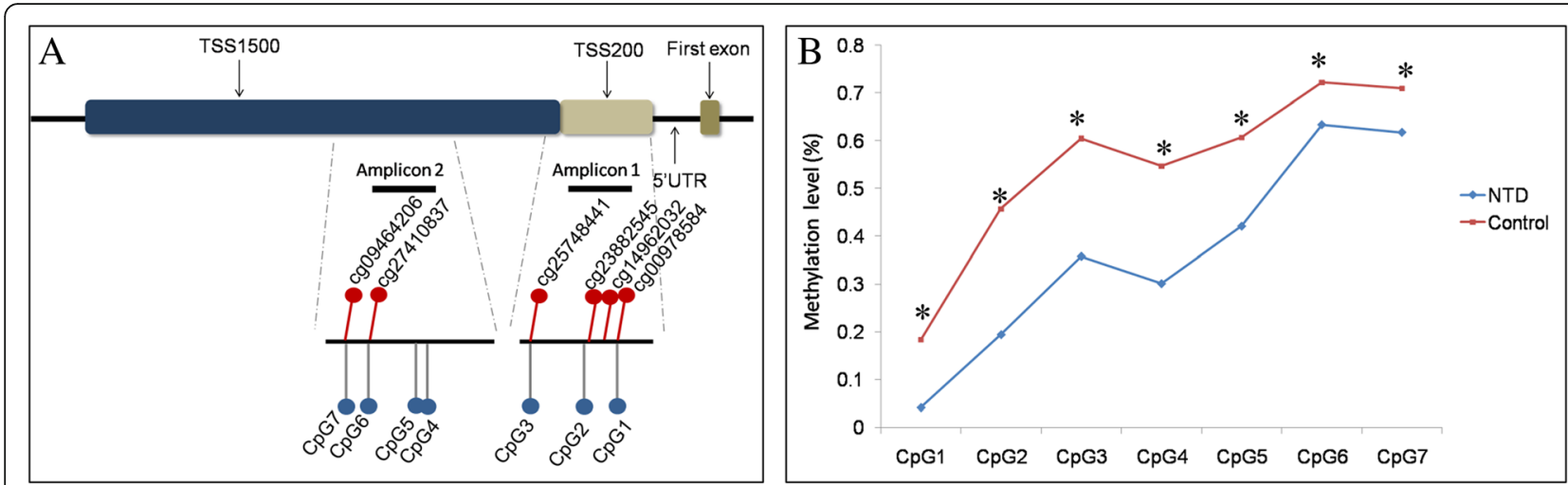

Fig. 1 Location and methylation levels of CpG sites in CASP8 examined in the discovery and validation stage in human subjects. a Locations of the CPG sites detected by HM450K and Sequenom EpiTYPER. The differentially methylated CpG units identified by HM450K Array are indicated as red dots above the black line. CpG sites detected in the validation stage are indicated as blue dots under the black line. b Methylation patterns of the CASP8 gene detected with Sequenom EpiTYPER in a larger sample of human subjects. Methylation levels for each CpG unit between NTD cases and controls within the amplicons were analyzed. HM450K, HumanMethylation450 BeadChip; NTD, neural tube defect. *Methylation levels of CpG sites were significantly different between the case and control groups (all $p<0.05$ )

the discovery stage. Amplicon 2 covered four CpG sites within the TSS1500 region of CASP8 (CpG4 to CpG7), in which CpG6 and CpG7 were the same as the cg27410837 and cg09464206 CpG sites, respectively, which were identified as differentially hypomethylated in the discovery stage.

All of the seven detected CpG sites were significantly hypomethylated in NTD cases, including three HM450K array-matched CpG sites (Fig. 1b). After Bonferroni correction, all of the sites demonstrated significant hypomethylation in NTD cases with a $\beta$ difference ranging between $8.8 \%$ and $26.3 \%$, reaffirming hypomethylated status in the promoter region of CASP8 in NTD cases. The details are shown in Additional file 1: Table S6.

The NTD group showed a significantly lower average methylation level of CASP8 (36.8\%) than the control group (54.7\%). Hypomethylation of CASP8 was a risk factor for NTDs (aOR $=1.11$; 95\% CI, 1.05-1.17) according to the logistic regression model, which indicated that the risk for NTDs increased by $10.8 \%$ with every $1 \%$ reduction of the average methylation level of CASP8. Risk for two major subtypes of NTDs, namely, anencephaly and spina bifida, was also associated with hypomethylated CASP8 (Table 1).

\section{PAH exposure and CASP8 methylation}

In a previous study, we found that levels of PAHs were significantly higher in maternal serum of NTD cases than in controls [6]. In the present study, we determined the correlation between the methylation status of CASP8 in fetal neural tissue and PAH levels in maternal serum. The methylation level of CASP8_CPG_2 showed an inverse correlation with total PAHs. Three CpG sites and the average methylation level of CASP 8 were inversely correlated with the concentration of H_PAHs. The remaining four $\mathrm{CpG}$ sites also tended to show an inverse correlation with H_PAHs, although statistical significance was not reached (Table 2). A subgroup analysis was made in NTD cases Additional file 1: Table S7; the results also indicated an inverse correlation between concentration of H_PAHs and CASP8 methylation in the NTD group.

\section{Oxidative stress and CASP8 methylation in human}

Correlation analysis of oxidative stress markers and the methylation status of CpG sites in CASP8 promoter in fetal neural tissues was performed. The methylation levels of several CpG sites were inversely correlated with antioxidant indicators GPx (CpG6, CpG7) and SOD (CpG7). One CpG

Table 1 Associations between mean methylation level of the CASP8 gene and risk of NTDs

\begin{tabular}{llllll}
\hline Group & $n$ & Mean methylation $(\% \pm$ SE) & $p$ value & OR $(95 \% \mathrm{Cl})$ & ${\text { Adjusted-OR }(95 \% \mathrm{Cl})^{a}}^{a}$ \\
\hline Total NTDs ${ }^{b}$ & 80 & $0.368(0.012)^{c}$ & $<0.001$ & $1.10(1.06,1.14)$ & $1.11(1.05,1.17)$ \\
Anencephaly & 33 & $0.381(0.018)$ & $<0.001$ & $1.08(1.04,1.13)$ & $1.11(1.03,1.19)$ \\
Spina bifida & 39 & $0.359(0.018)$ & $<0.001$ & $1.09(1.04,1.13)$ & $1.11(1.04,1.18)$ \\
Controls & 32 & $0.547(0.031)$ & - & 1.0 & 1.0 \\
\hline
\end{tabular}

Note: SE standard error, OR odds ratio, CI Confidence interval, NTDs neural tube defects

${ }^{a}$ Maternal general characteristics and exposure, including educational level, occupation, parity, unplanned pregnancy, and periconceptional folate

supplementation, which were unevenly distributed between the case and control groups, were adjusted in the logistic regression model

${ }^{b}$ Subtype analyses were not performed in eight encephalocele cases

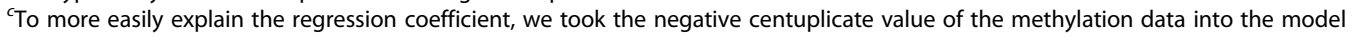


Table 2 Correlation analysis of differentially methylated CpG sites and PAH concentrations in maternal serum

\begin{tabular}{|c|c|c|c|c|c|c|c|}
\hline \multirow[t]{2}{*}{ CpG sites } & \multirow[t]{2}{*}{$N$} & \multicolumn{2}{|c|}{ Total PAHs, ng/g lipid } & \multicolumn{2}{|c|}{ L_PAHs, ng/g lipid } & \multicolumn{2}{|c|}{ H_PAHs, ng/g lipid } \\
\hline & & $\rho$ & $p$ & $\rho$ & $p$ & $\rho$ & $p$ \\
\hline CASP8_CpG_1 & 56 & -0.242 & 0.072 & -0.221 & 0.102 & -0.275 & 0.040 \\
\hline CASP8_CpG_2 & 58 & -0.275 & 0.037 & -0.257 & 0.051 & -0.290 & 0.027 \\
\hline CASP8_CpG_3 & 58 & -0.212 & 0.109 & -0.182 & 0.171 & -0.255 & 0.053 \\
\hline CASP8_CpG_4 & 58 & -0.250 & 0.058 & -0.212 & 0.111 & -0.296 & 0.024 \\
\hline CASP8_CpG_5 & 58 & -0.165 & 0.216 & -0.125 & 0.352 & -0.228 & 0.086 \\
\hline CASP8_CpG_6 & 58 & 0.011 & 0.937 & 0.061 & 0.650 & -0.101 & 0.450 \\
\hline CASP8_CpG_7 & 56 & -0.157 & 0.247 & -0.136 & 0.319 & -0.201 & 0.137 \\
\hline CASP8_average & 58 & -0.239 & 0.071 & -0.199 & 0.134 & -0.299 & 0.023 \\
\hline
\end{tabular}

Note: PAHs, polycyclic aromatic hydrocarbons; total PAHs, sum of all PAHs; L PAHs, sum of low-molecular-weight PAHs, including acenaphthylene, acenaphthene, fluorene, phenanthrene, anthracene, fluoranthene, and retene; $\mathrm{H}$ _PAHs, sum of high-molecular-weight PAHs, including pyrene, benz[a]anthracene, chrysene, benzo[b]fluoranthene, benzo[k]fluoranthene, and benzo[a]pyrene; $\rho$, Pearson's correlation coefficient.

site was inversely correlated with PC, a marker of protein oxidation (Table 3). A subgroup analysis was made in NTD cases (Additional file 1: Table S8), and the results indicated an inverse correlation between GPx (CpG6, CpG7, and CASP8_average) and CASP8 methylation in the NTD group.

\section{Casp8 methylation and expression in BaP-treated mouse embryos}

To validate the level of Casp 8 methylation in association with $\mathrm{BaP}$ exposure, a whole-embryo culture model was used. E8.5 embryos were explanted from the uterus of pregnant mice and cultured in rat serum with DMSO or BaP treatment. After $48 \mathrm{~h}$ of in vitro culture, the embryos developed into a stage where the neural tube would have closed under normal conditions. The embryos were collected and inspected under a dissecting microscope. The diameter of the yolk sac, the length of the head, and the crown-rump length of the mouse embryos cultured in $\mathrm{BaP}$ medium were smaller $(p<0.05)$ than those of the DMSO vehicle group (Additional file 2: Figure S1). Embryos treated with BaP showed a significantly higher rate of NTDs $(13.5 \%)$ than the vehicle group $(p<0.05$; Additional file 2: Figure S2 and Additional file 1: Table S9).
To examine whether CASP8 hypomethylation, which was detected in the promoter region of human NTD cases, was also present in BaP-treated mouse embryos, we amplified one DNA amplicon in the promoter of Casp 8 in mouse neural tissue. All of the five detected CpG sites were significantly hypomethylated in BaP-treated mouse embryos (Fig. 2). After Bonferroni correction, two $\mathrm{CpG}$ sites still demonstrated significant hypomethylation in the $\mathrm{BaP}$ group, with $\beta$ differences of $11.6 \%$ and $4.2 \%$, respectively, suggesting that decreased methylation of Casp 8 was present in BaP-treated mouse embryos which had increased incidence of NTDs. The details are represented in Additional file 1: Table S10.

Since hypomethylation is likely to induce gene upregulation, we analyzed the mRNA levels and protein abundance of caspase- 8 in neural tissue of BaP-treated mouse embryos. Consistent with decreased methylation levels in Casp 8 promoter, the mRNA expression of Casp 8 in the $\mathrm{BaP}$-treated group was significantly higher than that in DMSO controls, according to real-time PCR $(p<0.05$; Fig. 3a). Whole-mount in situ hybridization of Casp8 showed specific staining of neural tissues, particularly in the forebrain, midbrain, and hindbrain of BaP-treated E9.5

Table 3 Correlation analysis of oxidative stress markers in fetal neural tissues and differentially methylated CpG sites in CASP8

\begin{tabular}{|c|c|c|c|c|c|c|c|c|c|c|c|}
\hline \multirow[t]{2}{*}{ CpG sites } & \multirow[t]{2}{*}{$N$} & \multicolumn{2}{|c|}{ SOD, unit/mg protein } & \multicolumn{2}{|c|}{ GPx, unit/mg protein } & \multicolumn{2}{|c|}{ TAC, unit/mg protein } & \multicolumn{2}{|c|}{ MDA, nmol/mg protein } & \multicolumn{2}{|c|}{$\mathrm{PC}, \mathrm{nmol} / \mathrm{mg}$ protein } \\
\hline & & $\rho$ & $p$ & $\rho$ & $p$ & $\rho$ & $p$ & $\rho$ & $p$ & $\rho$ & $p$ \\
\hline CASP8_CpG_1 & 31 & -0.078 & 0.675 & -0.225 & 0.224 & 0.023 & 0.903 & 0.103 & 0.582 & -0.423 & 0.018 \\
\hline CASP8_CpG_2 & 31 & -0.005 & 0.980 & -0.091 & 0.627 & 0.038 & 0.840 & 0.093 & 0.618 & -0.266 & 0.148 \\
\hline CASP8_CpG_3 & 31 & -0.023 & 0.903 & -0.202 & 0.277 & 0.128 & 0.491 & 0.100 & 0.591 & -0.313 & 0.086 \\
\hline CASP8_CpG_4 & 31 & -0.128 & 0.493 & -0.186 & 0.317 & 0.022 & 0.907 & 0.073 & 0.696 & -0.256 & 0.164 \\
\hline CASP8_CpG_5 & 31 & -0.068 & 0.716 & -0.233 & 0.207 & 0.037 & 0.842 & 0.136 & 0.467 & -0.185 & 0.320 \\
\hline CASP8_CpG_6 & 31 & 0.006 & 0.976 & -0.402 & 0.025 & 0.144 & 0.440 & 0.183 & 0.323 & -0.029 & 0.875 \\
\hline CASP8_CpG_7 & 30 & -0.386 & 0.035 & -0.542 & 0.002 & 0.284 & 0.128 & 0.352 & 0.057 & -0.228 & 0.226 \\
\hline CASP8_average & 31 & -0.114 & 0.540 & -0.265 & 0.149 & 0.110 & 0.555 & 0.132 & 0.478 & -0.266 & 0.148 \\
\hline
\end{tabular}

Note: SOD superoxide dismutase, GPx glutathione peroxidase, TAC total antioxidant capacity, MDA malondialdehyde, $P C$ protein carbonyl, $\rho$ Pearson's correlation coefficient 


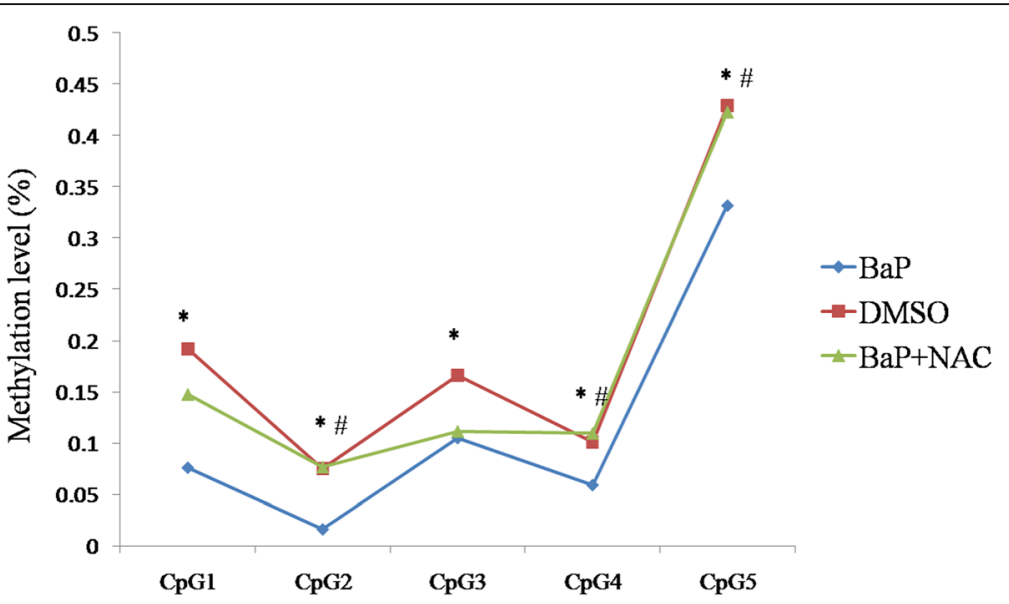

Fig. 2 Methylation levels of $\mathrm{CpG}$ sites in Casp8 examined in neural tissue of mouse embryos. The methylation levels for each CpG unit in the DMSO, BaP, and BaP + NAC groups within the amplicons were analyzed. DMSO, dimethylsulphoxide; BaP, benz(a)pyrene; NAC, N-acetyl-L-cysteine. ${ }^{*}$ Significant difference between DMSO and BaP group $(p<0.05)$. " Significant difference between BaP and BaP + NAC $(p<0.05)$

embryos (Fig. 3e). Western blot showed that the protein abundance of caspase-8 was much higher in BaP-treated embryos than in control embryos (Fig. $3 \mathrm{~b}$ and d). These results revealed upregulation of caspase- 8 in BaP-treated mouse embryos coupled with Casp 8 promoter hypomethylation.

\section{Effect of antioxidant on Casp8 methylation and expression}

To further examine the role that oxidative stress may have played in Casp 8 methylation during NTD formation, NAC, a known antioxidant, was added to $\mathrm{BaP}$ medium at the beginning of in vitro embryo culture.

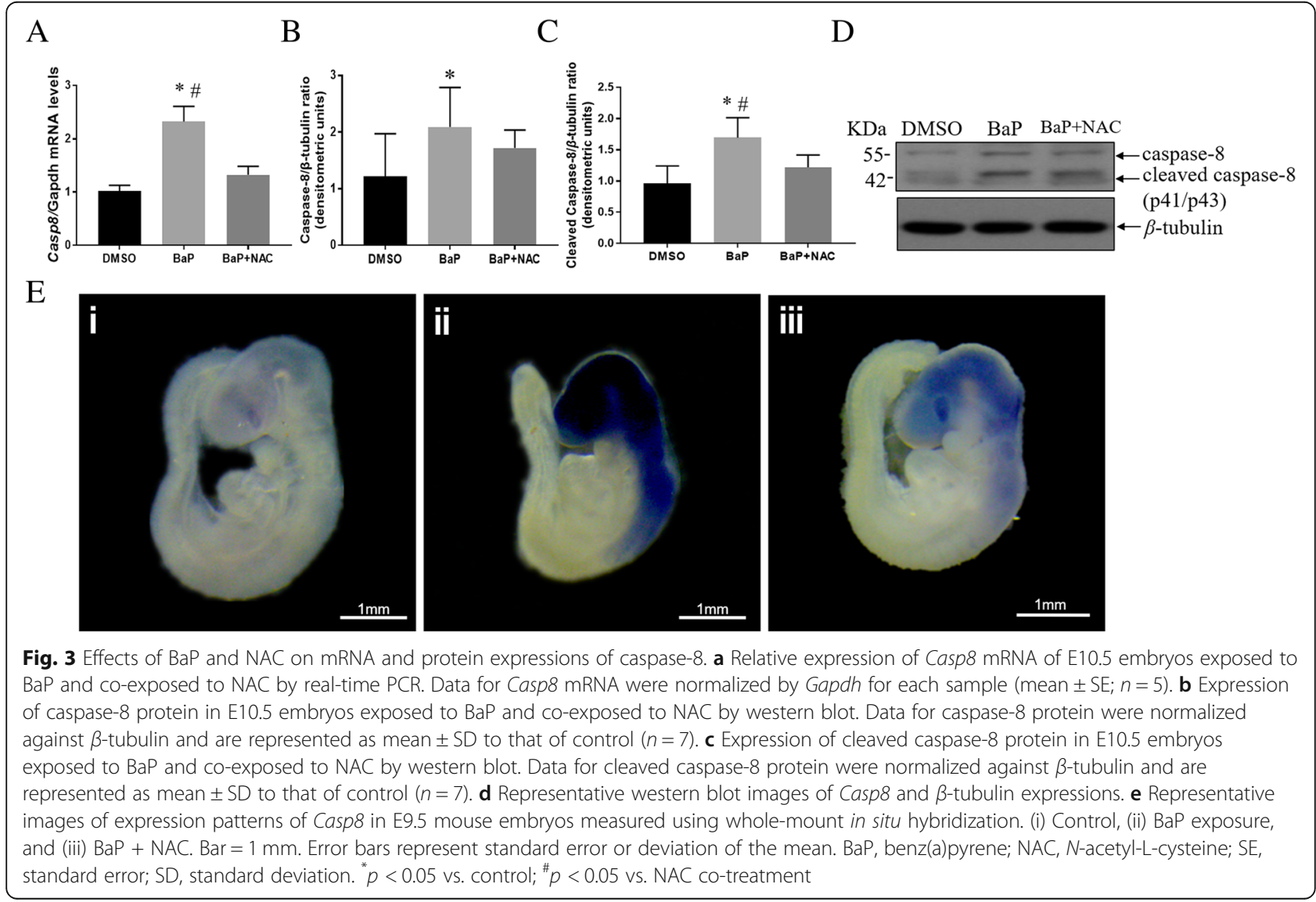


The diameter of the yolk sac, head length, and crownrump length all grew better in mouse embryos in the $\mathrm{NAC}$ and $\mathrm{BaP}$ co-treated group than those in the $\mathrm{BaP}$ group, with head length being restored to the same level as the vehicle group (Additional file 2: Figure S1). NAC rescued $\mathrm{BaP}$-induced NTDs in vitro $(p<0.05$; Additional file 1: Table S7).

We detected the methylation status of Casp 8 in mouse embryos co-treated by NAC and $\mathrm{BaP}$ to examine whether antioxidant could rescue the hypomethylation caused by $\mathrm{BaP}$. Three of the five $\mathrm{CpG}$ sites detected showed higher methylation levels in the group co-treated with NAC than in the BaP group (Fig. 2), one of which still showed significance after Bonferroni correction. These results suggested that Casp 8 hypomethylation caused by BaP could be rescued by antioxidation. More details are represented in Additional file 1: Table S8.

Caspase- 8 expression was also analyzed in the neural tissue of mouse embryos co-treated with NAC and BaP. The elevated mRNA expression of Casp 8 induced by $\mathrm{BaP}$ was normalized by NAC co-treatment $(p<0.05$; Fig. 3a and d). Consistent with the results of real-time PCR, NAC mitigated the expression of Casp 8 in the brain and spinal cord according to whole-mount in situ hybridization assays. Co-exposure to NAC also attenuated the increased procaspase- 8 protein expression induced by BaP exposure (Fig. $3 \mathrm{~b}$ and $\mathrm{d}$ ). Together, these findings revealed that antioxidation could rescue the upregulation of caspase- 8 accompanied by Casp 8 hypomethylation induced by $\mathrm{BaP}$ exposure.

\section{Effects of $\mathrm{BaP}$ and antioxidant on apoptosis}

Since caspase- 8 is thought to be the initiator caspase, we further hypothesized that overexpression of Casp 8 resulting from $\mathrm{BaP}$ exposure would lead to excessive apoptosis. Hence, we measured the protein level of cleaved caspase- 8 by western blot and detected apoptosis levels in embryos using TUNEL assays. Levels of cleaved caspase 8 were significantly upregulated after $\mathrm{BaP}$ exposure, while NAC treatment significantly suppressed BaP-induced caspase 8 cleavage (Fig. $3 \mathrm{c}$ and d). Apoptotic cells were more frequently observed in BaP-treated embryos than in controls $(p<0.05)$, while only a few TUNEL-positive cells were observed in mice co-treated with NAC and BaP as in the DMSO group (Fig. 4 and Additional file 2: Figure S3 and S4). These results suggest that antioxidation could alleviate apoptosis induced by BaP in mouse embryos.

\section{Discussion}

Increased caspase-8 expression and concomitant excessive apoptosis have been reported in PAH-related NTDs $[9,13]$, while the underlying mechanisms are not well studied. Under this context, we focused on the aberration of DNA methylation, one of the mechanisms behind gene-environment interaction. In this study, CASP8 hypomethylation was discovered in microarray data obtained from a small number of human NTD cases and controls and subsequently validated in a larger independent case-control cohort. The methylation levels of CASP8 were inversely correlated with concentrations of PAHs in maternal serum and with oxidative stress markers in fetal neural tissues. In further validation, increased NTD rates, Casp 8 hypomethylation, upregulation of caspase-8, increased cleaved caspase-8, and excessive apoptosis were found in BaPtreated mouse embryos cultured in an in vitro whole-embryo culture model. Antioxidant NAC attenuated the changes induced by $\mathrm{BaP}$ in mouse embryos.

Since NTD formation is thought to be associated with elevated activation of caspase- 8 , which plays a crucial role in the initiation of extrinsic apoptosis $[13,15]$, we detected the methylation level of CASP8 promoter in neural tissue from human NTD cases and controls. After exploring the HM450K microarray data and validating the CpG sites with a larger case-control cohort, we found that both average and locus-specific methylation levels in the promoter region of CASP8 were significantly decreased in human NTDs. According to a logistic regression model, the hypomethylation of CASP8 was a risk factor for NTDs $(\mathrm{aOR}=1.11$; 95\% CI, $1.05-$ 1.17), after adjusting for potential confounders. Generally, although not exclusively, hypomethylation of the gene promoter region is associated with gene upregulation [28]. Supportively, in our study, upregulated expression of mRNA and protein of caspase- 8 and increased cleaved caspase- 8 were found in mouse embryos that showed an increased incidence of NTDs accompanied with Casp 8 promoter hypomethylation in neural tissue after $\mathrm{BaP}$ exposure. The increased caspase- 8 and cleaved caspase- 8 found in our animal study are supported by previous studies that have found that elevated caspase-8/ cleaved caspase-8 levels in human fetuses are associated with increased risk for NTDs $[13,16]$. We further found elevated apoptosis levels in mouse embryos, which showed a decreased methylation level of Casp 8 promoter after $\mathrm{BaP}$ exposure. The deregulation of apoptosis has been implicated in the development of NTDs [2]. Although apoptosis is needed in the closure of the neural tube for the dorsolateral hinge points' formation and neural folds remodeling, excessive apoptosis may hinder the participation of cells in fusing neural folds or may disrupt the physical continuity of the dorsal midline, leading to NTDs [29]. To the best of our knowledge, no earlier studies have explored the role that aberrant DNA methylation of apoptosis-related genes may play in NTD formation, neither in human subjects nor in animals. These results suggest that Casp8 hypomethylation may induce excessive apoptosis by 

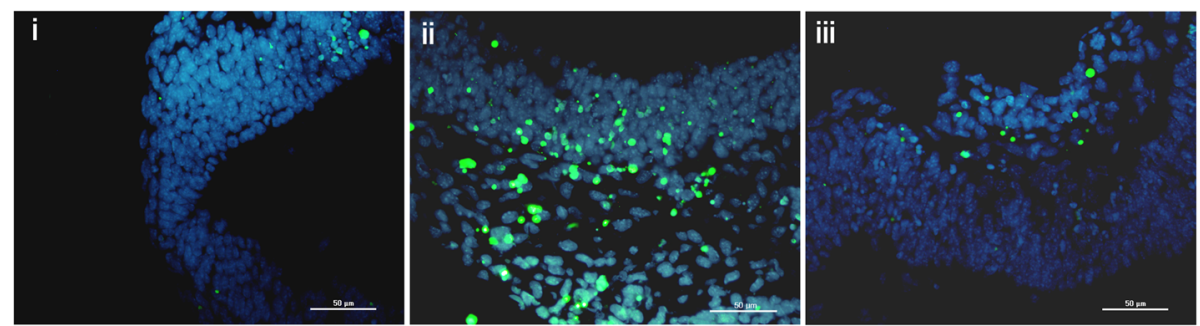

Fig. 4 Effects of BaP and NAC on apoptosis level of E10.5 embryos. Representative images of TUNEL-positive cells (green) and 4',6-diamidine-2phenylidoledihydrochloridestain cells (blue). Slices were sectioned along a coronal plane of mouse embryos. i Control, ii BaP exposure, and iii BaP + NAC. Bar $=50 \mu \mathrm{m}$. BaP, benz(a)pyrene; NAC, N-acetyl-L-cysteine

upregulating caspase-8 expression, leading to NTD formation. Our findings provide insight into the role of aberrant DNA methylation in abnormal neurulation during the development of human NTDs.

In previous studies, we found higher levels of cleaved caspase- 8 in NTD cases than in controls, and case mothers have higher levels of PAH exposure than control mothers [13]. Therefore, we analyzed the relationship between PAH levels in maternal serum and locus-specific methylation levels of CASP8 in neural tissue of NTD fetuses. The concentration of H_PAHs tended to be inversely correlated with all of the $\mathrm{CpG}$ sites and with the average methylation level of CASP8. To explore the association between PAH exposure and aberrant DNA methylation further, we treated mouse with $\mathrm{BaP}$ during neural tube closure. Significant lower locus-specific methylation levels were detected in the promoter of Casp 8 in neural tissue of mouse embryos in the BaP-treated group, which also showed an increased NTD rate.

Evidence regarding the effect of PAH exposure on Casp 8 methylation has not yet been retrieved. For global DNA methylation, the association between PAH exposure and decreased DNA methylation has been reported both in human and animal studies [19, 30, 31]. PAH metabolites in maternal urine are associated with decreased global methylation status in a Chinese cohort [32]. Several in vitro studies have found that $\mathrm{BaP}$ exposure could decrease global DNA methylation in a dose-response manner [33, 34]. However, the associations between PAH exposure and DNA methylation in specific genes were inconsistent in human, animal studies, and in vitro studies [11, 31, 35, 36]. For example, a case-control study conducted in China reported positive correlations between PAH levels in maternal serum and methylation levels of tight junction pathway genes CTNNA1 and MYH2 in neural tissue from NTD cases [11], while LINE-1 and MGMT genes were found to be hypomethylated in $82 \mathrm{PAH}$-exposed coke-oven workers compared to 62 unexposed controls, and gene-specific methylation of LINE-1 and MGMT was inversely correlated with levels of urinary PAH metabolites [31]. These studies indicate that PAHs may contribute to the methylation of distinct genes differently.

The mechanism by which PAH exposure causes aberrant DNA methylation during NTD formation remains unclear. Since oxidative stress has been implicated in the etiology of NTDs $[9,10,37]$ and has been reported to induce DNA hypomethylation in congenital anomalies $[23,38]$, we analyzed the associations between markers of oxidative stress and the methylation levels of locus-specific $\mathrm{CpG}$ sites in fetal neural tissues. Inverse correlations were found between antioxidant indicators or protein oxidation markers and the methylation status of CASP8 in NTD cases. To further explore the role of oxidative stress in BaP-induced DNA hypomethylation in NTD formation, we cultured mouse embryos in BaP with and without NAC. The incidence of NTDs was significantly lower in the NAC co-treated group than in the BaP-treated group. NAC also partially restored the decreased methylation level of Casp 8 to that of the control group. NAC is a kind of thiol and acts as a precursor in the synthesis of glutathione, which plays a vital role in cellular defense against oxidative stress. NAC has also been reported to ameliorate global DNA hypomethylation in arsenic-induced NTDs in chick embryos [38]. A randomized trial conducted in Vienna investigated the impact of an antioxidant- and vitamin-rich diet on the epigenetic pattern of $M L H 1$ in patients with noninsulin-dependent diabetes mellitus type 2 and impaired fasting glucose. In support of our study, this study found that antioxidant intervention increased the methylation level in promoter of $M L H 1$, and the DNA strand breaks induced by oxidative stress showed an inverse correlation with methylation level of $M L H 1$ [39].

Oxidative stress has been suggested as one of the possible mechanisms through which PAHs exert its effect on DNA methylation regulation [40]. A representative example of oxidative DNA damage, 8-hydroxy-2'-deoxyguanosine (8-OHdG), which is also increased in maternal serum in NTD cases [10], may suppress human DNA methyltransferase (DNMT) and murine Dnmt3a and inhibit DNA 
methylation by downregulating the methylation of nearby cytosine base [40, 41]. Moreover, oxidative stress may lead to the depletion of $S$-adenosyl-L-methionine (SAM), a methyl donor for methylation reaction under the catalysis of DNMT, which would reduce DNA methylation [42]. Further, increased ROS may also upregulate ten-eleven translocation (TET), an enzyme that induces demethylation, resulting in DNA hypomethylation [43]. The inverse correlation between oxidative markers and CASP8 methylation levels in human and the successful rescue of Casp 8 hypomethylation with NAC in mouse agrees with our hypothesis that PAHs may affect the methylation of CASP8/Casp 8 by inducing oxidative stress during NTD formation.

To the best of our knowledge, few studies have detected the methylation status of DNA directly in neural tissue of NTD cases. In consideration of the tissue specificity of DNA methylation, using central neural tissue from specific lesion sites is optimal in epigenetic studies of NTD etiology. However, the limitations of the present study should be mentioned. DNA methylation is time specific. The human tissues we collected were mostly at second trimester, while neural tube closure is completed at the end of the fourth-week post-fertilization, corresponding to about 6 weeks of clinical gestation. However, in our animal study, the neural tissue that we used to examine the methylation status was from mouse embryos that had just completed neural tube closure. In addition, we had no transcript-expression data for the CASP8 gene in human subjects since it is difficult to obtain tissues that are fresh enough from terminated NTD cases to extract mRNA. However, information on the expression of caspase- 8 from the in vitro mouse model allows insight from a different point of view. In addition, although the promoter region is the most studied region that is known to play a decisive role in the regulation of gene activity, DNA methylation function varies in different genomic contexts-for example, methylation in gene body may stimulate, but in TSS may block, transcription [44]. In this study, we only examined CpGs located in the promoter region and did not explore methylation changes in gene body and other regulatory regions. A different gene region should be taken into consideration in future studies.

\section{Conclusion}

Hypomethylation of the promoter in CASP8 is associated with an increased risk of NTDs in human subjects, and this association was confirmed in mice that were exposed to BaP. The decrease in the methylation status of CASP8/Casp 8 may be related to oxidative stress induced by $\mathrm{PAH}$ exposure. These findings provide novel insights into the role of epigenetic modifications of the Casp 8 in NTD formation in association with exposure to PAHs.

\section{Methods}

\section{Human subjects}

The enrollment of human subjects has been described in a previous report [5]. Briefly, human subjects were recruited from an ongoing population-based birth-defects surveillance system that was conducted in five rural counties in Shanxi Province of northern China between 2011 and 2014, where NTD prevalence is among the highest in the world, 31.5 per 10,000 births in 2014 [45]. For cases, fetuses electively terminated following prenatal diagnosis of an NTD were included. For controls, terminated fetuses with no congenital malformations were included. Information on sociodemographic characteristics, reproductive history, lifestyle, folic acid supplementation, active smoking and exposure to passive secondhand smoke, and fuel used for cooking and heating during the periconceptional period were collected through in-person interviews by trained local healthcare workers. Samples of maternal venous blood were collected at delivery or pregnancy termination, temporarily stored at $-20^{\circ} \mathrm{C}$ at local hospitals, and then transferred to our laboratory on dry ice and stored at $-80^{\circ} \mathrm{C}$ until use. Spinal cord and brain tissue samples were collected from terminated NTD and non-malformed fetuses by experienced pathologists and were immediately stored at $-80^{\circ} \mathrm{C}$ until the analyses were performed. The study protocol was approved by the Institutional Review Board of Peking University, and written informed consent was obtained from all participating women.

\section{Animals and whole-embryo culture}

Primigravida CD-1 (ICR) mice (ages 8-12 weeks) were used in the experiment. Female mice were mated with males at $8 \mathrm{AM}$, and vaginal plugs were examined $4 \mathrm{~h}$ later. $10 \mathrm{AM}$ on the day of finding a vaginal plug was considered 0 day of embryonic development (E0). Animals were cared for by the laboratory animal center at Peking University Health Science Center under a 12-h light/dark cycle in a temperature/humidity controlled facility and were allowed free access to food and water.

Whole-embryo culture was performed following the procedures described in a previous study [46]. Briefly, pregnant mice were sacrificed via cervical dislocation on E8.5. Only embryos with three to eight somites were utilized. Decidua and Reichert's membranes were removed before the embryos were randomly placed into sealed culture bottles (3-5 embryos per bottle) that contained rat serum with $1 \%$ dimethylsulphoxide (DMSO), $5 \mathrm{mM}$ $\mathrm{BaP}$ (Sigma-Aldrich), or $5 \mathrm{mM} \mathrm{N}$-acetyl-l-cysteine (NAC) and $5 \mathrm{mM} \mathrm{BaP}$, as determined by preliminary experiments. Then, the embryos were incubated at $37^{\circ} \mathrm{C}$ in sealed culture bottles fitted into a device that were rotated at $25 \mathrm{rpm}$. The culture bottles were initially gassed with a mixture of $5 \% \mathrm{O}_{2}, 5 \% \mathrm{CO}_{2}$, and $90 \% \mathrm{~N}_{2}$ for 2.5 
min. Subsequent gassing was performed after $16 \mathrm{~h}(20 \%$ $\mathrm{O}_{2}, 5 \% \mathrm{CO}_{2}$, and $\left.75 \% \mathrm{~N}_{2}\right)$ and $10 \mathrm{~h}\left(40 \% \mathrm{O}_{2}, 5 \% \mathrm{CO}_{2}\right.$, and $55 \% \mathrm{~N}_{2}$ ). Embryos used for ROS detection and whole-mount in situ hybridization were cultured for 24 $\mathrm{h}$ before being collected; the rest were cultured for $48 \mathrm{~h}$. Neural tissue was isolated from the embryos under a dissecting microscope and immediately put into liquid nitrogen before being stored at $-80^{\circ} \mathrm{C}$ for further use. The study protocol was approved by the Institutional Animal Care and Use Committee of Peking University (certificate no. LA2013-36).

\section{Methylation assay}

Methylation assays on DNA from human subjects were performed in two stages. In the first (i.e., the discovery stage), we used Infinium HumanMethylation450 BeadChip (HM450K; Illumina, San Diego, CA, USA) to profile genome-wide DNA methylation, using neural tissue from 10 NTD cases and 8 non-malformed control fetuses. In the second stage (i.e., the validation stage), neural tissues from 80 NTD cases and 32 non-malformed control fetuses were used to validate the results in the discovery stage by using the Sequenom MassARRAY system, as previously described elsewhere [11].

DNA was extracted from fetal neural tissue using the QIAamp DNA MiniKit (QIAGEN, Hilden, Germany). The concentration of DNA was measured with a NanoDrop2000 Ultramicro spectrophotometer (Thermo Fisher Scientific, MA, USA). All of the DNA samples were stored at $-80^{\circ} \mathrm{C}$ until used for assays. Bisulfite conversion of 500 ng DNA was conducted with an EZ DNA Methylation Kit (Zymo Research, CA, USA), following the manufacturer's instructions. To minimize the potential bias caused by variable conversion efficiency, the bisulfate conversion reaction was performed in duplicate for each sample, and the bisulfate-treated DNA was pooled for subsequent array analyses. Genome-wide DNA methylation was performed using the HM450K according to the manufacturer's protocol. Methylation array data for CASP8 were extracted from the genome-wide microarray.

In the validation stage, target verification of differentially methylated CpGs in the promoter of CASP8 was performed using the Sequenom EpiTYPER (Sequenom, San Diego, USA). Bisulfate-treated DNA was amplified via PCR. The PCR primers of CASP8 were designed using the online tool EpiDesigner (http://www.epidesigner.com/). We designed two amplicons to cover the six aberrant CpG sites, for which the differences between cases and controls were larger than 0.2 in the HM450K array. The primer sequence is presented in Additional file 1: Table S1). About $20 \mathrm{ng}$ of bisulfate-treated DNA was amplified by PCR. Enzyme digestion was performed using $\mathrm{T}$ cleavage enzyme (T Cleavage Mix) after the PCR product was incubated with shrimp alkaline phosphatase. Then, the product was desalinized using 384 dimple plates before being detected by the MassARRAY system. EpiTYPER Analyzer software version 1.0 was used to analyze the methylation levels of DNA. Positive controls (complete methylation) and negative controls (non-methylation) were used for quality control in methylation assay.

Mouse DNA methylation assay was also performed in Sequenom EpiTYPER (Sequenom, San Diego, USA). DNA was extracted from neural tissue of mouse embryos cultured with DMSO, BaP, or NAC and $\mathrm{BaP}$ co-treatment using the QIAamp DNA MiniKit (QIAGEN, Hilden, Germany). A total of $500 \mathrm{ng}$ genomic DNA from each sample was treated with bisulfite using the EZ DNA Methylation Kit (Zymo Research, CA, USA). Because Casp 8 may have multiple transcripts, to validate the differentially methylated $\mathrm{CpGs}$ found in the promoter of human CASP8 in mouse tissue, we designed amplicons within the promoter of mouse Casp 8 that regulate the exons that encode proteins sharing a high homology with the transcript discovered in the human methylation assay. PCR primer of Casp 8 was designed using the online tool EpiDesigner (http://www.epidesigner.com/). The primer sequence is presented in Additional file 1: Table S2.

\section{PAHs analysis}

The detailed methods for PAHs analysis have been described elsewhere [6]. Briefly, concentrations of PAHs in maternal serum were determined using an Agilent 7890A-5975C gas chromatograph and mass spectrometer equipped with an HP-5MS capillary column $(30 \mathrm{~m}$ $\times 0.25 \mathrm{~mm} \times 0.25 \mu \mathrm{m}$ ). Two procedure blanks and a reagent blank were included for each batch. Case-control status of the samples was masked during chemical analysis. PAH concentration is expressed on a lipid weight basis and is reported as nanogram per gram lipid. Low-molecular-weight PAHs (L_PAHs) with two or three benzene rings and high-molecular-weight PAHs (H_PAHs) with four or five benzene rings were used as sum respectively to indicate maternal PAH exposure.

\section{Oxidative stress evaluation}

Antioxidant indicators and oxidative damage markers in fetal neural tissues were determined according to the kit specifications (Nanjing Jiancheng Bioengineering Institute, Nanjing, China). Activity levels of superoxide dismutase (SOD), glutathione peroxidase (GPx), and total antioxidant capacity (TAC) were used as antioxidant indicators, and the contents of malondialdehyde (MDA) and protein carbonyl (PC) were used as indicators of lipid and protein oxidation, respectively. 


\section{Real-time PCR}

RNA was isolated from the neural tissue of E10.5 embryos using Trizol (Invitrogen). DNase I digestion (DNA-free, Ambion) was used to remove genomic DNA, after which RNA was reverse-transcribed using random hexamers (Superscript VILO cDNA synthesis kit). The abundance of Casp8 mRNA was analyzed using real-time PCR (iTaqTM Universal SYBR Green Supermix, BioRad) on a 7500 Fast Real Time PCR system (Applied Biosystems), with each sample analyzed in triplicate. The primer sequence is presented in Additional file 1: Table S3. The relative quantification of each level of gene expression was normalized according to Gapdh expression.

\section{Whole-mount in situ hybridization}

Whole-mount in situ hybridization of E9.5 mouse embryos was carried out using a protocol derived from previously published methods [47]. Briefly, Casp 8 probe was cloned by RT-PCR into pGEM-T (Promega) and used to generate digoxygenin-labeled cRNA probes by reverse transcription with T7 RNA polymerase (Roche). For detection, embryos were fixed in $4 \%$ paraformaldehyde at $4{ }^{\circ} \mathrm{C}$ overnight before being dehydrated through a graded series of methanol/PBT $(25 \%, 50 \%, 75 \%$, and $100 \%$ methanol in PBST) and stored at $-20^{\circ} \mathrm{C}$ for future detection. After rehydration, the embryos were incubated in bleached 4:1 PBST:30\% hydrogen peroxide for $1 \mathrm{~h}$ on ice before being digested in $10 \mu \mathrm{g} / \mathrm{mL}$ proteinase $\mathrm{K}$. Then the preheated hybridization buffer was used to prehybridize the embryos for $1 \mathrm{~h}$ at $70^{\circ} \mathrm{C}$. After that, embryos were hybridized overnight in $1 \mu \mathrm{g} / \mathrm{mL}$ DIG-labeled RNA probe for Casp 8 and washed with Solution I (50\% formamide, $5 \times$ SSC pH 5, 1\% SDS) and Solution II $(0.5 \mathrm{M} \mathrm{NaCl}, 10 \mathrm{mM}$ Tris $\mathrm{HCl} \mathrm{pH} 7.5,0.1 \%$ Tween 20) the next day at $70{ }^{\circ} \mathrm{C}$. Next, the embryos were pre-blocked in $10 \%$ sheep serum before being incubated in anti-Digoxigenin-AP antibody (1:2000, Roche) in 1\% sheep serum overnight at $4{ }^{\circ} \mathrm{C}$. Color detection was carried out using a developing solution (NBT/BCIP, Roche) in the dark, and the signal was captured periodically via a dissecting microscope.

\section{Western blot}

Protein was extracted on ice from embryos at E10.5 using RIPA buffer. Protein concentrations were determined using the Bradford assay. Western blot was performed using conventional methods, with $50-\mu \mathrm{g}$ protein run per sample on NuPAGE $4-12 \%$ Bis-Tris gel (Life technologies), followed by transfer to PVDF membranes (XCell II Blot Module, Invitrogen). The primary antibodies were rabbit anti-caspase-8 (1:500, Proteintech, Chicago, USA) and anti- $\beta$-TUBULIN $(1: 10,000$, Proteintech, Chicago, USA). After incubation with secondary antibody (1:10,000, DAKO), blots were developed using ECL Prime (GE Healthcare Life Sciences) or ECL Western Blotting Substrate (Promega). ImageJ was used to detect densitometry. The results were normalized using $\beta$-TUBULIN loading control. Independent experiments were carried out three to four times per sample.

\section{TUNEL assay}

Paraffin slices of E10.5 mouse embryos were used for apoptotic cell injury assays using the one-step TUNEL kit according to the manufacturer's instruction (Beyotime Institute of Biotechnology). Briefly, the slices were dewaxed with xylene before being incubated with proteinase $\mathrm{K}$, followed by TUNEL reaction agents for $1 \mathrm{~h}$ at $37^{\circ} \mathrm{C}$. Under a microscope (at $\times 400$ magnification), cells with green fluorescence were defined as apoptotic cells. Five fields were randomly selected from each section, and all cells were successively counted for each field. The ratio of the number of TUNEL-positive cells to the total number of cells was determined.

\section{Statistical analysis}

During the discovery stage, Illumina Genome Studio software (Illumina, CA, USA) was used to extract signal intensities for each probe, perform initial quality-control checks, and estimate $\beta$-scores and detection $p$ values. The $\beta$-score was defined as the proportion of total signal from the methylation-specific probe or color channel. The detection $p$ value was calculated as $1-p$ value computed from the background model characterizing the chance that the target sequence signal could be distinguished from the negative controls. Independent $t$ tests were used to identify differentially methylated CpG sites of CASP8 between cases and controls. Correction for multiple comparisons was performed using the Bonferroni procedure. Differentially methylated CpG sites were identified by the following criteria: false discovery rate $p<0.05$ and absolute $\beta$-value difference $>0.2$.

Within the validation stage, the $\chi^{2}$ test or Fisher's exact test was used to compare demographic information between the case and control groups. The difference in methylation intensity of CASP8 between cases and controls was tested using the Shapiro-Wilk test. The independent $t$ test was used to identify differentially methylated CpG sites in CASP8. The association between the methylation levels of CpG sites in CASP8 and risk of NTDs was estimated from the odds ratio (OR) with 95\% confidence interval (CI), using an unconditional logistic model. To better explain the coefficient, we used the negative centuplicate $\beta$-score in the model. We adjusted for general maternal characteristics and exposure, including educational level, occupation, parity, unplanned pregnancy, and periconceptional folate supplementation, which were unevenly distributed between 
the case and control groups. Correlation analyses of differentially methylated CpG sites and PAH levels in maternal serum as well as oxidative stress markers in fetal neural tissues were performed by Pearson's correlation coefficient.

In the mouse study, the rate of NTDs was analyzed using Pearson's $\chi^{2}$ test. Differentially methylated CpG sites of Casp8 in neural tissues among fetal mice with DMSO, BaP, and NAC and BaP co-treatment were detected by one-way analysis of variance (ANOVA). The abundance of mRNA and protein and the levels of apoptosis was expressed as mean \pm SE (SD) and were analyzed by ANOVA followed by LSD (equal variances assumed) or Dunnett's T3 (equal variances not assumed). A two-tailed $p$ value of $<0.05$ was considered statistically significant. Statistical analyses were conducted using SPSS 23.0 (IBM Co., NY, USA).

\section{Additional files}

Additional file 1: Table S1. The PCR primer sequences in Sequenom EpiTYPER sequencing for human. Table S2. The PCR primer sequences in Sequenom EpiTYPER sequencing for mouse. Table S3. The sequences of primer for real-time PCR. Table S4. Methylation of CASP8 gene using the Human Methylation 450 Bead Chip assay. Table S5. Demographic and obstetric characteristics of NTD cases and controls in Shanxi Province, China, 2011-2014. Table S6. Validation of differentially methylated CpG sites of CASP8 gene in neural tissues of NTD cases and controls with Sequenom EpiTYPER. Table S7. Correlation analysis of differentially methylated $\mathrm{CpG}$ sites and PAH concentrations in maternal serum in NTD cases. Table S8. Correlation analysis of oxidative stress markers in fetal neural tissues and differentially methylated $\mathrm{CpG}$ sites in CASP8 in NTD cases. Table S9. Embryotoxicity of BaP and the effect of NAC in mouse embryo. Table S10. Differentially methylated CpG sites in Casp8 in neural tissues of mouse embryo with and without BaP exposure/NAC rescue. (DOCX $65 \mathrm{~kb}$ )

Additional file 2: Figure S1. Head and crown-rump lengths of E10.5 mouse embryos exposed to BaP or BaP and NAC. Figure S2. NTDs in E10.5 mouse embryos exposed to BaP in vitro. Figure S3. The effects of $\mathrm{BaP}$ and NAC on apoptosis level of E10.5 embryos. Figure S4. Slice of E10.5 mouse embryo. (DOCX $1781 \mathrm{~kb})$

\section{Abbreviations}

8-OHdG: 8-hydroxy-2'-deoxyguanosine; ad-p: Adjusted $p$ value; ANOVA: Oneway analysis of variance; BaP: Benz(a)pyrene; CASP8/Casp8: Caspase-8; Chr: Chromosome; Cl: 95\% confidence interval; DMSO: Dimethylsulphoxide; DNMT: DNA methyltransferase; E: Embryonic day; Fb: Forebrain; GPx: Glutathione peroxidase; H_PAHs: High-molecular-weight PAHs; Hb: Hindbrain; HM450K: Infinium HumanMethylation450 BeadChip; L PAHs: Low-molecular-weight PAHs; Mb: Midbrain; MDA: Malondialdehyde; NAC: N-acetyl-L-cysteine; NTDs: Neural tube defects;"; OR: Odds ratio; PAHs: Polycyclic aromatic hydrocarbons; PC: Protein carbonyl; ROS: Reactive oxygen species; SAM: S-adenosyl-L-methionine; SOD: Superoxide dismutase; TAC: Total antioxidant capacity; TET: Ten-eleven translocation; UTR: Untranslated region

\section{Acknowledgements}

We would like to thank the local healthcare workers in the maternal and child health centers of Taigu, Pingding, Xiyang, Shouyang, and Zezhou in Shanxi Province, China, for their help with patient recruitment and data collection.

\section{Funding}

This study was supported in part by the National Key Research and Development Program, Ministry of Science and Technology, the Peoples' Republic of China (Grant No. 2016YFC1000501), and grants from the National Natural Science Foundation of China (Grant No. 31371523 and 81773441) and the Ministry of Education of China (Grant No. 20130001110064).

\section{Availability of data and materials}

Not applicable.

\section{Authors' contributions}

YH contributed to the study design, performed the experiments, conducted the statistical analyses, and drafted the manuscript. SSL contributed to the study design and animal model and critically revised the manuscript. $L$ and ZWL contributed to the study design, recruited the study patients, and critically revised the manuscript. JAM critically revised the manuscript. AGR and LLW conceived and supervised the project and critically revised the manuscript. All authors read and approved the final manuscript.

\section{Ethics approval and consent to participate}

For human subjects, the study protocol was approved by the Institutional Review Board of Peking University, and written informed consent was obtained from all participating women.

All mice were maintained at the laboratory animal center at Peking University Health Science Center using standard techniques in accordance with protocols approved by the Institutional Animal Care and Use Committee of Peking University (certificate no. LA2013-36).

\section{Consent for publication}

Not applicable.

\section{Competing interests}

The authors declare that they have no competing interests.

\section{Publisher's Note}

Springer Nature remains neutral with regard to jurisdictional claims in published maps and institutional affiliations.

\section{Author details}

${ }^{1}$ Institute of Reproductive and Child Health, National Health Commission Key Laboratory of Reproductive Health, Peking University, Beijing 100191, China. ${ }^{2}$ Department of Epidemiology and Biostatistics, School of Public Health, Peking University, Beijing 100191, China. ${ }^{3}$ Division of Birth Cohort Study, Guangzhou Women and Children's Medical Center, Guangzhou Medical University, Guangzhou 510623, China. ${ }^{4}$ Department of Epidemiology, Mailman School of Public Health, Columbia University Medical Center, 722 West 168th Street, New York, NY 10032, USA. ${ }^{5}$ Herbert Irving Comprehensive Cancer Center, Columbia University Medical Center, New York, NY 10032 , USA.

Received: 13 January 2019 Accepted: 26 April 2019

Published online: 07 May 2019

\section{References}

1. Wallingford JB, Niswander LA, Shaw GM, et al. The continuing challenge of understanding, preventing, and treating neural tube defects. Science (New York, NY). 2013;339(6123):1222002 PMID: 23449594

2. Copp AJ, Stanier P, Greene ND. Neural tube defects: recent advances, unsolved questions, and controversies. The Lancet Neurology. 2013;12(8): 799-810 PMID: 23790957; eng.

3. Ren AG. Prevention of neural tube defects with folic acid: the Chinese experience. World journal of clinical pediatrics. 2015;4(3):41-4 PMID: 26261765; eng.

4. Christianson A, Howson C, Modell B. March of Dimes global report on birth defects: the hidden toll of dying and disabled children [cited 2018 May 25]. Available from: https://www.marchofdimes.org/materials/global-report-onbirth-defects-the-hidden-toll-of-dying-and-disabled-children-full-report.pdf

5. Ren A, Qiu X, Jin $L$, et al. Association of selected persistent organic pollutants in the placenta with the risk of neural tube defects. Proceedings of the National Academy of Sciences of the United States of America. 2011; 108(31):12770-5 PMID: 21768370; eng. 
6. Wang $B$, Jin L, Ren A, et al. Levels of polycyclic aromatic hydrocarbons in maternal serum and risk of neural tube defects in offspring. Environmental science \& technology. 2015;49(1):588-96 PMID: 25488567; eng.

7. Yi D, Yuan $Y$, Jin $L$, et al. Levels of PAH-DNA adducts in cord blood and cord tissue and the risk of fetal neural tube defects in a Chinese population. Neurotoxicology. 2015;46:73-8 PMID: 25522656; eng.

8. Yuan $Y$, Jin L, Wang $L$, et al. Levels of PAH-DNA adducts in placental tissue and the risk of fetal neural tube defects in a Chinese population. Reproductive toxicology (Elmsford, NY). 2013;37:70-5 PMID: 23416326; eng.

9. Lin $S$, Ren A, Wang $L$, et al. Oxidative stress and apoptosis in benzo[a]pyrene-induced neural tube defects. Free radical biology \& medicine. 2018;116:149-58 PMID: 29309894; eng.

10. Yuan $Y$, Zhang $L$, Jin $L$, et al. Markers of macromolecular oxidative damage in maternal serum and risk of neural tube defects in offspring. Free radical biology \& medicine. 2015;80:27-32 PMID: 25542138; eng

11. Wang $L$, Lin S, Zhang J, et al. Fetal DNA hypermethylation in tight junction pathway is associated with neural tube defects: A genome-wide DNA methylation analysis. Epigenetics. 2017;12(2):157-65 PMID: 28059605.

12. Greene ND, Stanier P, Copp AJ. Genetics of human neural tube defects. Human molecular genetics. 2009;18(R2):R113-29 PMID: 19808787; eng.

13. Wang L, Lin S, Yi D, et al. Apoptosis, expression of PAX3 and P53, and caspase signal in fetuses with neural tube defects. Birth defects research Part A, Clinical and molecular teratology. 2017;109(19):1596-604 PMID: 28786179.

14. Dong D, Reece EA, Yang P. The Nrf2 activator vinylsulfone reduces high glucose-induced neural tube defects by suppressing cellular stress and apoptosis. Reproductive sciences. 2016; PMID: 26802109.

15. Tummers B, Green DR. Caspase-8: regulating life and death. Immunological reviews. 2017;277(1):76-89 PMID: 28462525.

16. Yang P, Li X, Xu C, et al. Maternal hyperglycemia activates an ASK1-FoxO3acaspase 8 pathway that leads to embryonic neural tube defects. Science signaling. 2013;6(290):ra74 PMID: 23982205; eng.

17. Das DN, Panda PK, Mukhopadhyay S, et al. Prediction and validation of apoptosis through cytochrome P450 activation by benzo[a]pyrene. Chemicobiological interactions. Feb. 2014;5(208):8-17. PMID: 24239969; eng.

18. Henkler F, Stolpmann K, Luch A. Exposure to polycyclic aromatic hydrocarbons: bulky DNA adducts and cellular responses. Exs. 2012;101:10731 PMID: 22945568; eng.

19. Herbstman JB, Tang D, Zhu D, et al. Prenatal exposure to polycyclic aromatic hydrocarbons, benzo[a]pyrene-DNA adducts, and genomic DNA methylation in cord blood. Environmental health perspectives. 2012;120(5): 733-8 PMID: 22256332; eng.

20. Tang WY, Levin L, Talaska $G$, et al. Maternal exposure to polycyclic aromatic hydrocarbons and 5'-CpG methylation of interferon-gamma in cord white blood cells. Environmental health perspectives. 2012;120(8):1195-200 PMID: 22562770; eng.

21. Wang X, Guan Z, Chen Y, et al. Genomic DNA hypomethylation is associated with neural tube defects induced by methotrexate inhibition of folate metabolism. PloS one. 2015;10(3):e0121869 PMID: 25822193; Eng.

22. Fan $R$, Wang $D$, Mao $C$, et al. Preliminary study of children's exposure to PAHs and its association with 8-hydroxy-2'-deoxyguanosine in Guangzhou, China. Environment international. 2012;42:53-8 PMID: 21511339; eng.

23. Udomsinprasert W, Kitkumthorn N, Mutirangura A, et al. Global methylation, oxidative stress, and relative telomere length in biliary atresia patients. Scientific reports. 2016;6:26969 PMID: 27243754; eng.

24. Zhu Y, Liao X, Lu L, et al. Maternal dietary zinc supplementation enhances the epigenetic-activated antioxidant ability of chick embryos from maternal normal and high temperatures. Oncotarget. 2017;8(12):19814-24 PMID: 28177898; eng.

25. Lan CC, Huang SM, Wu CS, et al. High-glucose environment increased thrombospondin-1 expression in keratinocytes via DNA hypomethylation. Transl Res. 2016;169:91-101.e1-3 PMID: 26678678; eng.

26. Zhang $X$, Hadley $C$, Jackson IL, et al. Hypo-CpG methylation controls PTEN expression and cell apoptosis in irradiated lung. Free radical research. 2016; 50(8):875-86 PMID: 27367846; eng.

27. Das DN, Panda PK, Naik PP, et al. Phytotherapeutic approach: a new hope for polycyclic aromatic hydrocarbons induced cellular disorders, autophagic and apoptotic cell death. Toxicology mechanisms and methods. 2017;27(1): 1-17 PMID: 27919191; eng.

28. Deaton AM, Bird A. CpG islands and the regulation of transcription. Genes \& development. 2011;25(10):1010-22 PMID: 21576262; eng.
29. Greene ND, Copp AJ. The embryonic basis of neural tube defects. New York: Oxford University Press; 2006. eng. (DF W, editor. Neural tube defects: from origin to treatment).

30. Kuc C, Richard DJ, Johnson S, et al. Rainbow trout exposed to benzo[a]pyrene yields conserved microRNA binding sites in DNA methyltransferases across 500 million years of evolution. Scientific reports. 2017;7(1):16843 PMID: 29203905; eng.

31. Duan $\mathrm{H}, \mathrm{He} \mathrm{Z}, \mathrm{Ma}$ J, et al. Global and MGMT promoter hypomethylation independently associated with genomic instability of lymphocytes in subjects exposed to high-dose polycyclic aromatic hydrocarbon. Archives of toxicology. 2013;87(11):2013-22 PMID: 23543013; eng.

32. Yang P, Gong YJ, Cao WC, et al. Prenatal urinary polycyclic aromatic hydrocarbon metabolites, global DNA methylation in cord blood, and birth outcomes: A cohort study in China. Environmental pollution (Barking, Essex: 1987). 2018;234:396-405 PMID: 29202418; eng.

33. Minero AS, Lukashevich OV, Cherepanova NA, et al. Probing murine methyltransfease Dnmt3a interactions with benzo[a]pyrene-modified DNA by fluorescence methods. The FEBS journal. 2012;279(20):3965-80 PMID: 22913541; eng.

34. Wilson $\mathrm{VL}$, Jones PA. Inhibition of DNA methylation by chemical carcinogens in vitro. Cell. 1983;32(1):239-46 PMID: 6825170; eng.

35. Gao D, Lin J, Ou K, et al. Embryonic exposure to benzo(a)pyrene inhibits reproductive capability in adult female zebrafish and correlation with DNA methylation. Environmental pollution (Barking, Essex : 1987). 2018;240:40311 PMID: 29753248; eng

36. Jiang $\mathrm{CL}$, He SW, Zhang YD, et al. Air pollution and DNA methylation alterations in lung cancer: A systematic and comparative study. Oncotarget. 2017;8(1):1369-91 PMID: 27901495; eng.

37. Tung EW, Winn LM. Valproic acid increases formation of reactive oxygen species and induces apoptosis in postimplantation embryos: a role for oxidative stress in valproic acid-induced neural tube defects. Molecular pharmacology. 2011;80(6):979-87 PMID: 21868484

38. Han ZJ, Song G, Cui Y, et al. Oxidative stress is implicated in arsenic-induced neural tube defects in chick embryos. Int J Dev Neurosci. 2011;29(7):673-80 PMID: 21723934; Eng.

39. Switzeny OJ, Mullner E, Wagner KH, et al. Vitamin and antioxidant rich diet increases MLH1 promoter DNA methylation in DMT2 subjects. Clinical epigenetics. 2012;4(1):19 PMID: 23025454; eng.

40. Teneng I, Montoya-Durango DE, Quertermous $\mathrm{J}$, et al. Reactivation of L1 retrotransposon by benzo(a)pyrene involves complex genetic and epigenetic regulation. Epigenetics. 2011;6(3):355-67 PMID: 21150308; eng.

41. Weitzman SA, Turk PW, Milkowski DH, et al. Free radical adducts induce alterations in DNA cytosine methylation. Proceedings of the National Academy of Sciences of the United States of America. 1994;91(4):1261-4 PMID: 8108398.

42. Hitchler MJ, Domann FE. An epigenetic perspective on the free radical theory of development. Free radical biology \& medicine. 2007;43(7):1023-36 PMID: 17761298; eng.

43. Chia N, Wang L, Lu X, et al. Hypothesis: environmental regulation of 5hydroxymethylcytosine by oxidative stress. Epigenetics. 2011;6(7):853-6 PMID: 21617369; eng.

44. Jones PA. Functions of DNA methylation: islands, start sites, gene bodies and beyond. Nature reviews Genetics. 2012;13(7):484-92 PMID: 22641018.

45. Liu J, Zhang L, Li Z, et al. Prevalence and trend of neural tube defects in five counties in Shanxi province of Northern China, 2000 to 2014. Birth defects research Part A, Clinical and molecular teratology. 2016; PMID: 26879384; Eng.

46. New DA. Whole-embryo culture and the study of mammalian embryos during organogenesis. Biological reviews of the Cambridge Philosophical Society. 1978;53(1):81-122 PMID: 352414.

47. Kyryachenko S, Kyrylkova K, Leid M, et al. Determination of gene expression patterns by whole-mount in situ hybridization. Methods in molecular biology (Clifton, NJ). 2012;887:15-22 PMID: 22566042; eng. 